\title{
Intramuscular haemodynamics using near infra-red spectroscopy as a research strategy to understand chronic muscle pain pathophysiology
}

\author{
Takuo Kuboki ${ }^{\mathrm{a}, *}$, Kenji Maekawa ${ }^{\mathrm{a}}$ and Glenn Clark ${ }^{\mathrm{b}}$ \\ ${ }^{a}$ Department of Oral and Maxillofacial Rehabilitation, Okayama University Graduate School of \\ Medicine and Dentistry, Okayama, Japan \\ ${ }^{\mathrm{b}}$ Division of Diagnostic Science, USC School of Dentistry, CA, USA
}

\begin{abstract}
Recently, localized intramuscular haemodynamic disturbance has been recognized as one of the cardinal features of a chronically painful muscle. To test this hypothesis, we have carried out several studies that have assessed intramuscular haemodynamics to understand more about haemodynamic alteration under conditions of an experimentally induced vasoreactive challenges in healthy controls and in patients with chronic muscle pain using near infra-red (NIR) spectroscopy. In addition, we have validated the NIR based haemodynamic signals by comparing against a water signal intensity change taken from a transverse $\mathrm{T}_{2}$-weighted magnetic resonance image (MRI) of the muscles. As a result, we discovered that muscle pain patients have a diminished ability to vasodilate in painful muscle. We also provided powerful evidences that NIR data (total haemoglobin level), transcutaneously taken from the muscles in humans could be an analogue of intramuscular haemodynamics.
\end{abstract}

\section{Introduction}

Chronic muscle pain is known to be highly prevalent and it can induce substantial daily life disability in humans $[1,2]$. Although significant effort has been devoted to determining its pathophysiology, the exact mechanisms have not been firmly established. In recent years, localized intramuscular haemodynamic disturbance has been recognized as one of the cardinal features of a chronically painful muscle. This diminished vascular response might be involved only a secondary phenomena of the pain inducing mechanism or it be a essential aspect of the primary process causing pain [3]. For these reasons, measuring haemodynamics precisely in skeletal muscle proper is one important goal of muscle pain researchers. While several devices have been used previously, they have substantial shortcomings (invasive, measuring area is too small, etc.). To try to solve this dilemma, we have carried out several studies that have assessed intramuscular haemodynamics in the masseter and trapezius muscles using near infra-red (NIR) spectroscopy. This work was designed to understand more about haemodynamic alteration under conditions of an experimentally induced vasoreactive challenge in healthy controls and

\footnotetext{
${ }^{*}$ Corresponding author: Dr. Takuo Kuboki, Department of Oral and Maxillofacial Rehabilitation, Okayama University Graduate School of Medicine and Dentistry, 2-5-1 Shikata-cho, Okayama 700-8525, Japan. Tel.: +81 86235 6681; Fax: +81 86235 6684; E-mail: kuboki@md.okayama-u.ac.jp.
} 
in patients with chronic muscle pain. Herein, we summarize our research and discuss the benefits and limitation of NIR-based-signal monitoring of masseter and trapezius muscle haemodynamics in humans.

\section{NIR spectroscopy device}

\subsection{Principle}

The near infra-red spectroscopy photometer we utilized was RunMan CWS-2000 (NIM Inc., Philadelphia, USA). This device monitors intramuscular haemodynamics by dual wavelength techniques, which employed characteristics of the haemoglobin isosbestic point reported by Chance (1951) [4]. This pioneering work demonstrated that there are two distinctly different absorption spectra for oxyhaemoglobin and deoxyhaemoglobin because the former has absorbance at $760 \mathrm{~nm}$ while the later shows absorbance at $850 \mathrm{~nm}$. The opposite occurs when deoxyhaemoglobin is re-oxygenated. Alterations in the total haemoglobin blood concentration in the illuminated area affect both wavelengths similarly and results in a baseline shifts in both curves. The RunMan device is based on this optical technology and it capitalizes on the above described differences in absorption characteristics of these two haemoglobin molecules. Light is only a small part of the electromagnetic spectrum, which spans the range from radio waves to light to X-rays and beyond. The light band in turn is divided into three major segments: infra-red, visible and ultraviolet. The RunMan technique is based on light in the near infra-red range (i.e. between infra-red and visible). Near infra-red light has a unique ability to penetrate biological tissues. However, the interaction of light with biological tissues is complex. A single wavelength of light has varying transmission characteristics through tissue in the same way that visible light interacts with stained glass. Furthermore, the various colors or wavelengths of light interact differently with biological tissues. Certain tissues are transparent at specific wavelengths, while others are completely opaque at certain wavelengths. Using this theory, the RunMan unit has been used to measure muscle deoxygenation during exercise and changes in brain oxygenation during hypoxic stress or ischaemia $[5,6]$.

\subsection{Photometer probe}

The RunMan probe is designed to emit light into a tissue. The light is scattered randomly within the tissue and some is transmitted back to the photodetectors. A broad range of wavelengths is emitted by the tungsten filament lamps in the probe. Many wavelengths are absorbed by the outer layers of skin while others pass freely through these layers. The light which transverse the epidermal layers strikes inner tissues such as muscle. Bone, which has similar index of refraction to that of tissue in the NIR range, has a minimal effect on light transport. Once the NIR light reaches the denser internal tissues, it is scattered and largely absorbed. Absorption of NIR light is dependent upon physiological conditions in the tissue. As we have mentioned, the $760 \mathrm{~nm}$ and $850 \mathrm{~nm}$ wavelengths utilized by the RunMan instrument are characteristic for the two forms of the haemoglobin molecule. This device does not provide actual blood volume measurements since only a portion of the incident light eventually reaches the photodetectors. All wavelengths except $760 \mathrm{~nm}$ and $850 \mathrm{~nm}$ are filtered out, leaving only the light intensity changes due to haemoglobin. When the two signals $(760 \mathrm{~nm}$ and $850 \mathrm{~nm}$ ) are added together, this combined signal represents the total haemoglobin blood concentration in the illuminated area. In our studies we utilized only the combined signal (760 and $850 \mathrm{~nm}$ ) and our underlying assumption is that the total hemoglobin signal is an analog of total blood volume. 
While it is clear that the magnitude of the signal is dependent upon the paths of the originally incident photons, or 'particles' of light, the RunMan probe we used was engineered for a photon depth penetration of 2.5 centimeters. This was possible because light, which illuminates the sample can penetrate only to shallow depths and theories describing photon migration are only applicable over relatively short distances. Actual photon pathlength, however, is random walk- or diffusion-like patterns rather than straight paths. The resulting photon migration patterns exhibit a wide variety of paths from a source to detector distributed between a straight line in the subcutaneous fat layers beneath the skin and a circuitous route scattered to depths of several centimeters where the light is largely absorbed. Thus the probable paths exhibit a banana-shaped distribution and the device therefore has significant detection capabilities in a volume approximately $2 \mathrm{~cm}$ wide by $2-3 \mathrm{~cm}$ deep.

\section{Intramuscular NIR signal responses to physiological vasoactivation}

In one of our first NIR based studies, we utilized controlled sustained isometric contraction of the jaw closers to alter blood flow in the masseter and temporalis muscles. Before, during, and after these sustained muscle contractions we monitored combined 760 and $850 \mathrm{~nm}$ intramuscular NIR signals from these muscles [7]. By recording this signal across time, we could determine changes in the intramuscular blood volume and assess the effect of low levels $(5,15,25$ and 100\%) of maximum voluntary contraction (MVC). Twenty subjects, 10 males (mean age: $30.5 \pm 5.6$ ) and 10 females (mean age: $27.6 \pm 4.8$ ), without pain or dysfunction in the masticatory system were included in this study. Data were recorded for 30 seconds before, 30 seconds during and 5 minutes after the 4 sustained contraction tasks. The results showed that all four levels of voluntary contraction produced a clear haemodynamic response (Fig. 1). Sustained muscle contraction induces a decrease in intramuscular blood volume during contraction and a clear rebound increase in blood volume after cessation of the contraction. Repeated measure ANOVA revealed a significant main effect for the different contraction levels (during contraction: $p=0.001$, after contraction: $p<0.001)$. These data are in general agreement with previous reports that have shown that skeletal muscle contraction reduces blood volume due to mechanical compression of the blood vessels [8], and after the contraction, rebound vasodilation (post-contraction hyperaemia) is induced by metabolic, neurogenic, and blood-borne vasodilative factors.

Next, we elected to use cold pressor stimulation (ice water immersion) of either the hand or foot region as a method of altering hemodynamics. This model had advantages in that the subjects were not required to perform a difficult sustain contraction task, but instead simply sit still while we monitored the changes in the NIR signal changes in resting muscles [9]. Cold pressor stimulation (CPS) involves a sustained immersion of a limb in ice water and is well known to induce systemic sympathetic nerve activity increase. Nine healthy non-smoking male subjects (mean age: $24.0 \pm 1.9$ ) without a history of chronic muscle pain or vascular headaches participated. Their right hand was immersed in a water bath $\left(4,10,15^{\circ} \mathrm{C}\right)$ for exactly 1 minute. Each trial lasted 7 minutes ( 1 minute before, 1 minute during, 5 minutes after) and a strict random order was utilized for the 3 test temperatures and the mock trial. Again, we elected to record blood volume changes from the masseter muscle but in this experiment we also recorded heart rate and blood pressure levels across the experimental period. Results showed that blood volume increased progressively during CPS as the water temperature decreased $(p<0.0001)$ and very little effect was seen during the mock trial (Fig. 2). Heart rate and blood pressure levels also increased progressively during CPS as the temperature decreased.

Assuming a non-specific sympathetic response was triggered by CPS, our data would indicate that a beta adrenergic response (vasodilatory) predominated over any vasoconstrictive response within the 


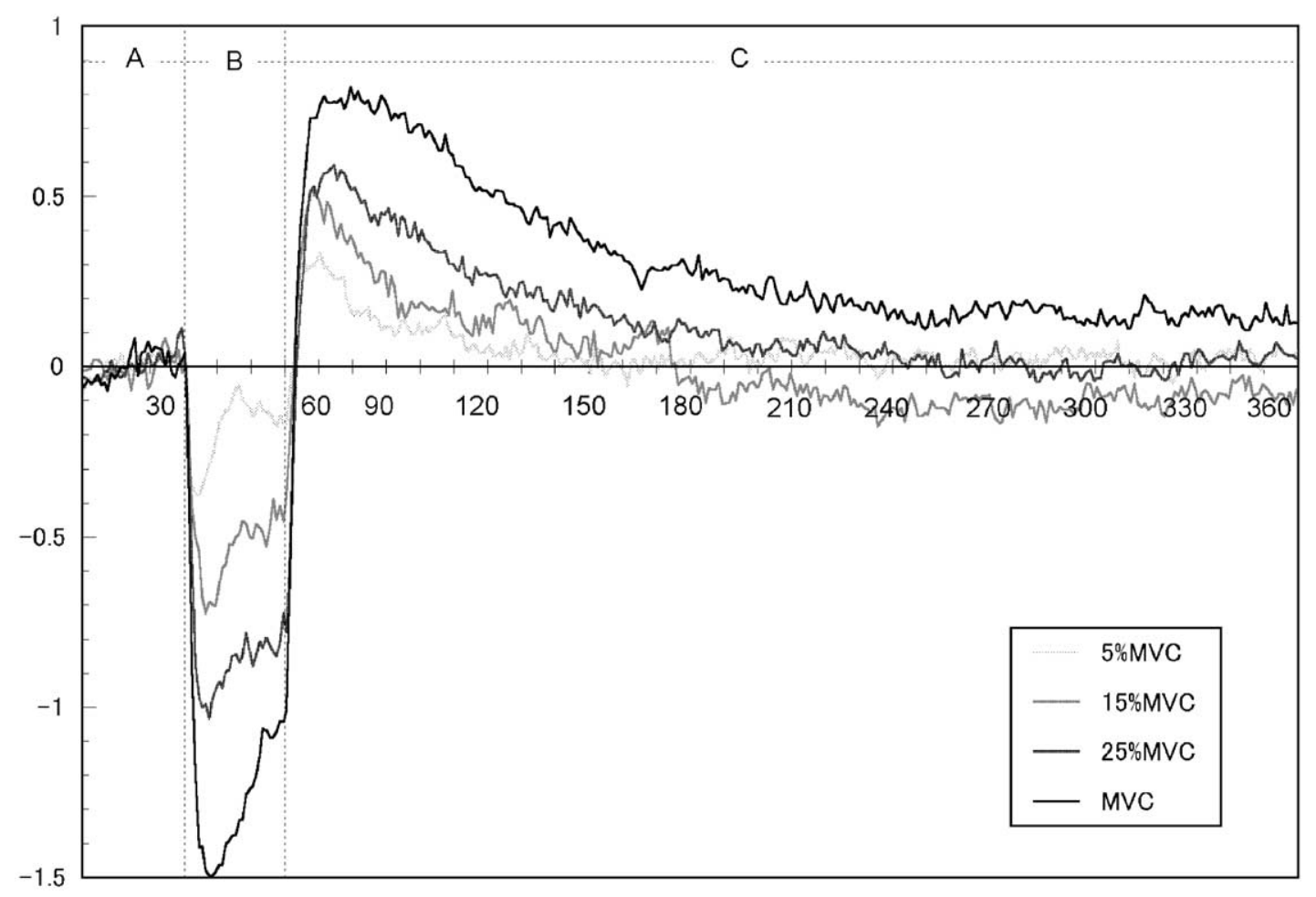

Fig. 1. Mean normalized blood volume (Hb level) changes seen in two relevant phases (contraction and recovery) at 5,15 , 25 , and 100\% maximum voluntary clenching (MVC) trials in masseter muscle. This study evaluated the influence of low level contraction forces on intramuscular haemodynamics in human masseter muscle using near infrared tissue spectroscopy. Twenty subjects, 10 males and 10 females, without pain or dysfunction in the masticatory system were included in this study. Data were recorded for 30 seconds before, 30 seconds during and 5 minutes after the 4 sustained contraction tasks. The results showed that all four levels of voluntary contraction produced a clear haemodynamic response (during and after contraction) in masseter muscle. Repeated measure analysis of variance revealed a significant main effect for the different contraction levels in masseter muscle (during contraction: $p=0.001$, after contraction: $p<0.001$ ). A: Baseline readings before the experimental contraction recorded for 30 seconds; B: readings during an isometric voluntary contraction recorded for 30 seconds; C: readings during the recovery phase recorded for 240 seconds after contraction. Y axis indicates averaged normalized rate of the readings relative to the maximum change after contraction at $100 \%$ MVC trial (1.0). According to [7].

masseter muscle. This conclusion is reasonably supported by basic research studies that have suggested that beta adrenergic receptors are more abundant within intramuscular vessels than other vasoactive receptors that might induce vasoconstrictive effect (alpha-adrenergic, neuropeptide Y, etc.) [10-12]. In addition, since beta receptors are also abundant in heart, the observed cardiovascular activity increase during CPS is logical also. Overall, the observed strong increase in intramuscular blood volume in the masseter muscles during CPS applied to the hand-forearm is presumed to be due to both a local vasodilative response within the muscle and increased cardiac output.

The results of above two studies are interesting, but one limitation of NIR spectroscopy is that the exact path of the reflected light through the illuminated tissue and the absolute blood volume can be estimated but not precisely known. Another concern that exists for any transcutaneus physiologic signal measuring device, is that the contribution of the cutaneous tissues to the resultant signal is also unknown. For these reasons, we conducted another set of studies to determine how precisely NIR spectroscopy can detect the intramuscular haemodynamics. 


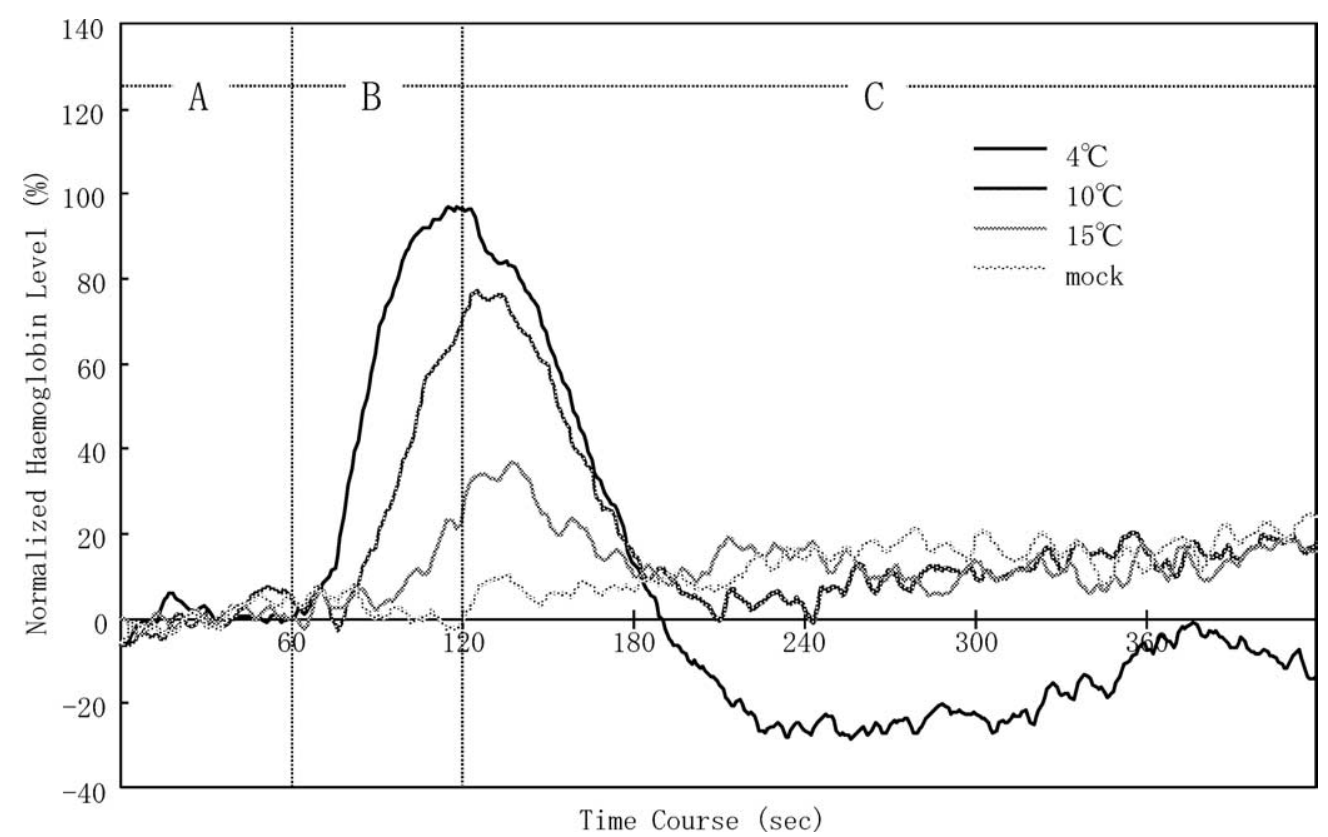

Fig. 2. Cold pressor stimulus (CPS) temperature and resting masseter muscle haemodynamics in normal human subjects. CPS is a well-known sympathetic activator of the skeletal muscles. The effect of CPS (sympathetic overactivation) on the resting haemodynamics of the right masseter muscle in normal subjects was determined by using near infra-red spectroscopy. Nine healthy non-smoking male subjects without a history of chronic muscle pain or vascular headaches participated and their right hand was immersed in a water bath $\left(4,10,15^{\circ} \mathrm{C}\right)$ for exactly 1 minute. Haemoglobin levels increased progressively during CPS as the water temperature decreased $(p<0.0001)$ and very little effect was seen during the mock trial. A: Baseline $(1$ minute), B: during CPS (1 minute), C: recovery phase (5 minutes). According to [9].

\section{Validity test of NIR spectroscopy for measuring intramuscular haemodynamics}

We elected to compare and contrast the combined 760-850 NIR signal change against a water signal intensity change taken from a transverse $\mathrm{T}_{2}$-weighted magnetic resonance image of the muscle under two different conditions (voluntary contraction and cold pressor stimulation). The $\mathrm{T}_{2}$-weighted MRI signal does not measure blood volume directly, however it does measure tissue-specific water content changes. The underlying assumption is that a rapid change the water content of the imaged tissue would be due to an alteration in the blood volume changes since water is the predominate component of blood. Of course we cannot determine if the fluids changes being monitored were intravascular, extravascular or intracellular. Nevertheless, the primary advantage of this technique was that the targeted deep tissue can be isolated from other tissues on the 3D image created by the MRI. Moreover, the area that is targeted for analysis can also be precisely defined.

Other researchers support the opinion that the $\mathrm{T}_{2}$-weighted MRI method gives a signal output that is highly reflective of blood volume changes in the muscle tissue [13-15]. For example, several MRI researchers have looked at the post-exercise changes on the signal intensity of $T_{2}$-weighted MRI in skeletal tissues [16-21]. As would be expected, these investigations uniformly show that the $T_{2}$ signal intensity increases immediately after the cessation of exercise, presumably due to post-exercise hyperaemia. These studies all show that $\mathrm{T}_{2}$-weighted MRI is a useful tool for analysis of dynamic changes in intramuscular blood volume. 


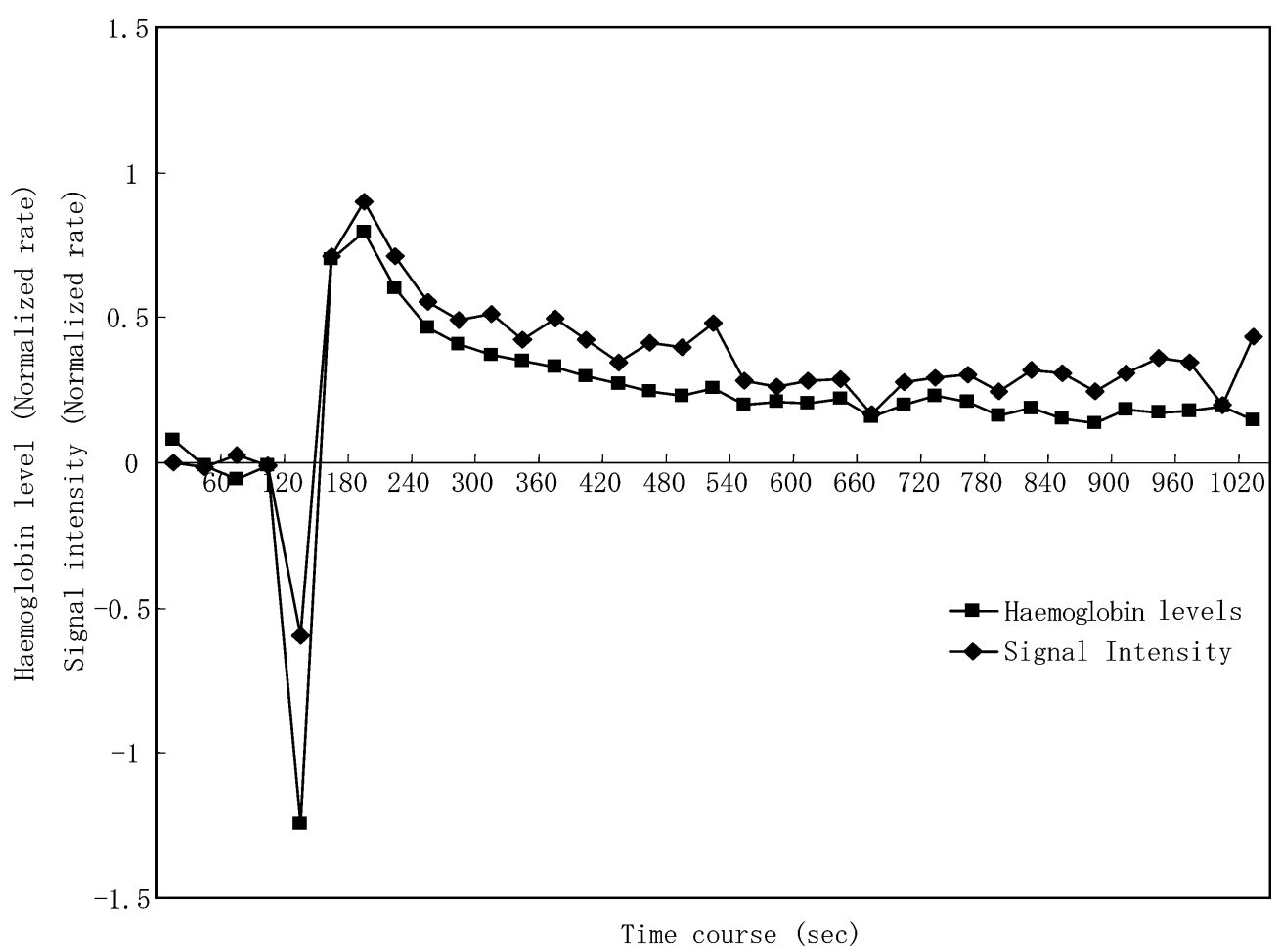

Fig. 3. Near-infrared spectroscopy signals and signal intensity in $T_{2}$-weighted MR imaging of the human masseter muscle. This study compared blood volume changes transcutaneously measured using near-infrared spectroscopy against water signal intensity changes taken from a transverse $\mathrm{T}_{2}$-weighted MR image of the masseter muscle in healthy human subjects before, during and after contraction. Eight healthy non-smoking males with no history of chronic muscle pain or vascular headaches participated (mean age: $23.9 \pm 0.6$ years). Intramuscular haemoglobin levels and water content of the right masseter muscle were continuously monitored for 2 minutes before, 30 seconds during and 15 minutes after a maximum voluntary clenching (MVC) task. Plots of the data showed that both NIR-based total $\mathrm{Hb}$ and $\mathrm{T}_{2}$-weighted MRI-based signal-intensity levels clearly decreased during MVC and a clear post-contraction rebound response was evident after the MVC. The NIR data were found to be highly correlated to MRI-based signal-intensity data (Pearson's $r=0.945, p<0.0001$ ). According to [22].

Our first NIR-MRI comparison study involved collecting data from normal human subjects. First we collected the combined 760/850 NIR signal of the masseter muscle before, during and after a $100 \%$ maximum voluntary contraction sustained for 30 seconds. Next, these same subjects were placed in an MRI imaging tube and the $\mathrm{T}_{2}$-weighted MRI signal was monitored before during and after this same clenching task [22]. This study involved 8 healthy non-smoking males (mean age: $23.9 \pm 0.6$ ) with no history of chronic muscle pain or vascular headaches. The MRI data were gathered using a turbo spin echo sequence (TR: $2300 \mathrm{msec}$; TE: $90 \mathrm{msec}$; FOV: $188 \times 300 \mathrm{~mm}$; scanning time: $30 \mathrm{sec}$; slice thickness: $10 \mathrm{~mm}$ ) and the slice level was set at mid-point between the origin and insertion of the masseter. Intramuscular blood volume and water content of the right masseter muscle were continuously monitored for 2 minutes before, 30 seconds during and 15 minutes after a 100\% maximum voluntary clenching (MVC) task. Both the NIR and MRI data were baseline-corrected and normalized and mean levels were established and plotted. Plots of the data showed that both NIR-based blood volume and $\mathrm{T}_{2^{-}}$ weighted MRI-based signal-intensity levels clearly decreased during MVC and a clear post-contraction rebound response was evident after the MVC (Fig. 3). The NIR data were found to be highly correlated to MRI-based signal-intensity data (Pearson's $r=0.945, p<0.0001$ ). These data provide powerful 
evidence that NIR data (total haemoglobin level), transcutaneously taken from the masseter muscle in humans, well reflects the intramuscular water signal intensity changes seen using a $\mathrm{T}_{2}$-weighted MRI imaging method.

One problem with this experiment was that the imaging methods (NIR and MRI) were not performed simultaneously, but at different times. This was done because of the deleterious effect the MRI's magnetic field has on the NIR probe and amplifier. Another problem was that the muscle shape changes during the contraction itself and we were unsure how much effect the anatomic alteration would have on the signal itself. The first problem can be solved by having the subject simply practice the task to achieve consistency. To solve second problem, we elected to use a cold pressor stimulation as the method of inducing vasoactivation. This allowed us to examine the MRI-T $\mathrm{T}_{2}$ signal changes in a resting muscle where presumably the shape of the muscle was unchanged during the experiment. The experiment involved taking $\mathrm{T}_{2}$-weighted MR images taken from the human trapezius muscle before, during, and after CPS. Fifteen healthy male volunteers (mean age 25.9 \pm 2.1 ) participated in this study [23]. $\mathrm{T}_{2}$-weighted MRI was acquired using a 1.5 tesla MR unit with a body array coil. The MRI data was collected using a turbo spin echo sequence (TR: $2000 \mathrm{msec}$, TE: $99 \mathrm{msec}$, FOV: $225 \times 450 \mathrm{~mm}$, scanning time: $26 \mathrm{sec}$, slice thickness: $10 \mathrm{~mm}$ ). The slice level was set perpendicular to the muscle long axis at the mid-point of the horizontal portion of the right trapezius muscle. CPS $\left(4^{\circ} \mathrm{C}\right)$ was applied to each subject's right foot and ankle for 2 minutes. The signal intensity changes were recorded continuously for 7 minutes before, 2 minutes during, and 6 minutes after withdrawal of CPS. Six of these subjects also underwent a mock-CPS trial. Each participant's MRI-based signal intensity data was standardized using the average of 7 minute baseline data and then analyzed statistically. The mean signal intensity level in $\mathrm{T}_{2}$-weighted trapezius muscle MRI significantly increased during CPS $(p<0.0001$, one way repeated measure ANOVA) and rapidly returned to the baseline level after cold pressor withdrawal (Fig. 4). No statistically significant signal changes were observed across the mock-CPS trial subjects. These findings are identical to cold pressor-induced haemodynamic changes documented in the trapezius muscle by NIR spectroscopy evaluation in a different experiment in our laboratory [24]. A comparison of the averaged MRI-T $\mathrm{T}_{2}$ signal data from the study with that from NIR based study revealed high correlation (Pearson's $r=0.805, p<0.0001$ ) between the two data sets.

One interesting finding is that the time to baseline recovery seen in the masseter muscle with contraction induce vasodilation was more prolonged (5 to 10 minutes) than we observed in the trapezius muscle (1-2 minutes). Admittedly the muscle were different and this might explain the differences but we also could speculate that a contraction induce vasodilation produces more inherent interstitial edema while a CPS vasodilation produces a more reactive vasodilation and not interstitial edema.

\section{Intramuscular haemodynamics in chronic localized muscle pain}

Based on the above findings, we utilized NIR spectroscopy to assess vasoreactive changes in chronic localized muscle pain patients [24]. The study involved submitting nine controls (mean age: 24.6 years), who had no medical history of migraine, hypertension or sustained pain in the trapezius region and nine chronic trapezius muscle pain subjects (mean age: 23.2 years) to CPS stimulation. All subjects were males. The result was that, in the muscle pain subjects, the intramuscular Hb level changes during CPS were significantly reduced compared to normal controls (Fig. 5). However, heart rate and blood pressure changes did not show a statistical difference between two groups. One feasible explanation for the diminished response to CPS in the muscle pain subjects is that these individuals have an impaired 


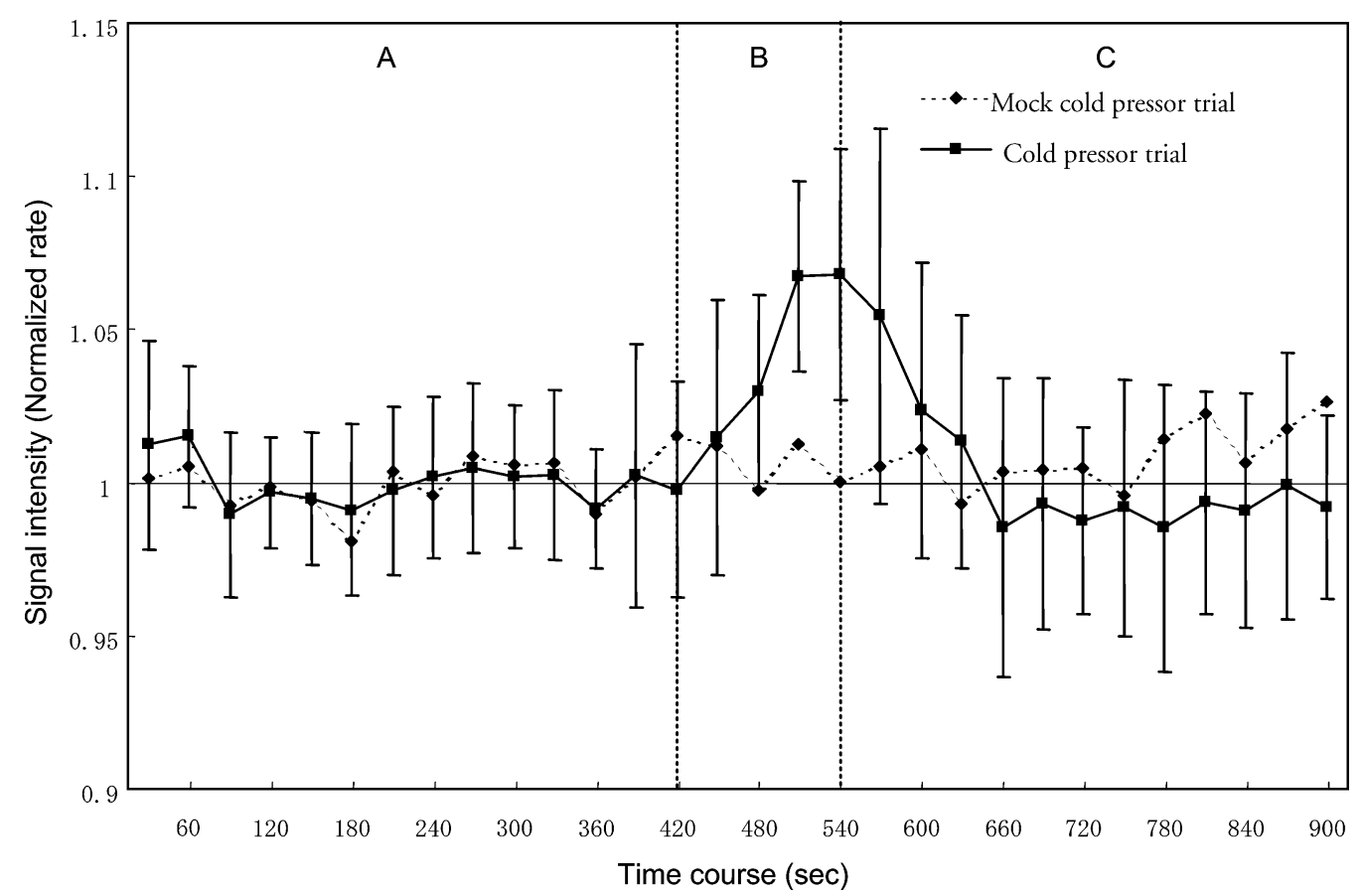

Fig. 4. Effect of cold pressor stimulation on signal intensity changes in $T_{2}$-weighted $M R$ imaging of the human trapezius muscle. This study evaluated the ability of signal intensity (SI) in $\mathrm{T}_{2}$-weighted trapezius muscle magnetic resonance imaging (MRI) to detect intramuscular haemodynamic changes during cold pressor stimulation (CPS). Fifteen healthy volunteers (mean age, $25.9 \pm 2.1$ years) participated in this study. $\mathrm{T}_{2}$-weighted MRI was acquired using a 1.5 tesla MR unit with a body array coil. Cold pressor stimulation $\left(4^{\circ} \mathrm{C}\right)$ was applied to each subject's right foot and ankle for 2 minutes. The SI changes were recorded continuously for 7 minutes before, 2 minutes during, and 6 minutes after withdrawal of cold pressor stimulation. The mean SI level in $\mathrm{T}_{2}$-weighted trapezius muscle MRI significantly increased during CPS $(p<0001$, one way repeated measure ANOVA and post hoc contrast analysis) and returned to the baseline level after cold pressor withdrawal. These findings are identical to the cold pressor-induced haemodynamic changes documented in the trapezius muscle by NIR spectroscopy evaluation [24]. A: Baseline before cold pressor stimulation, B: during cold pressor stimulation, C: recovery period after cold pressor stimulation. According to [23].

ability to vasodilate their intramuscular vascular beds. We need to push forward further research to make clear the cause of this impairment but at this point we are looking at the possibility of an endogenous desensitization of beta-adrenergic receptors.

\section{Conclusions}

We conclude that NIR spectroscopy can detect intramuscular haemodynamics precisely upon physiological vasoactive stimulation in humans. We know that low levels of sustained jaw muscle contraction (as low as 5\% MVC) can produce a relative ischaemic state in the muscle. Using this equipment, we discovered that muscle pain patients have a diminished ability to vasodilate in painful muscle. We next need to evaluate the haemodynamic changes in the non-painful muscles of patients who have localized chronic muscle pain to find out if this diminished vasodilatory response is generalized to all muscles or just those that are painful. Also further research with biological assessment should be done to clarify the mechanisms of this vascular alteration. 


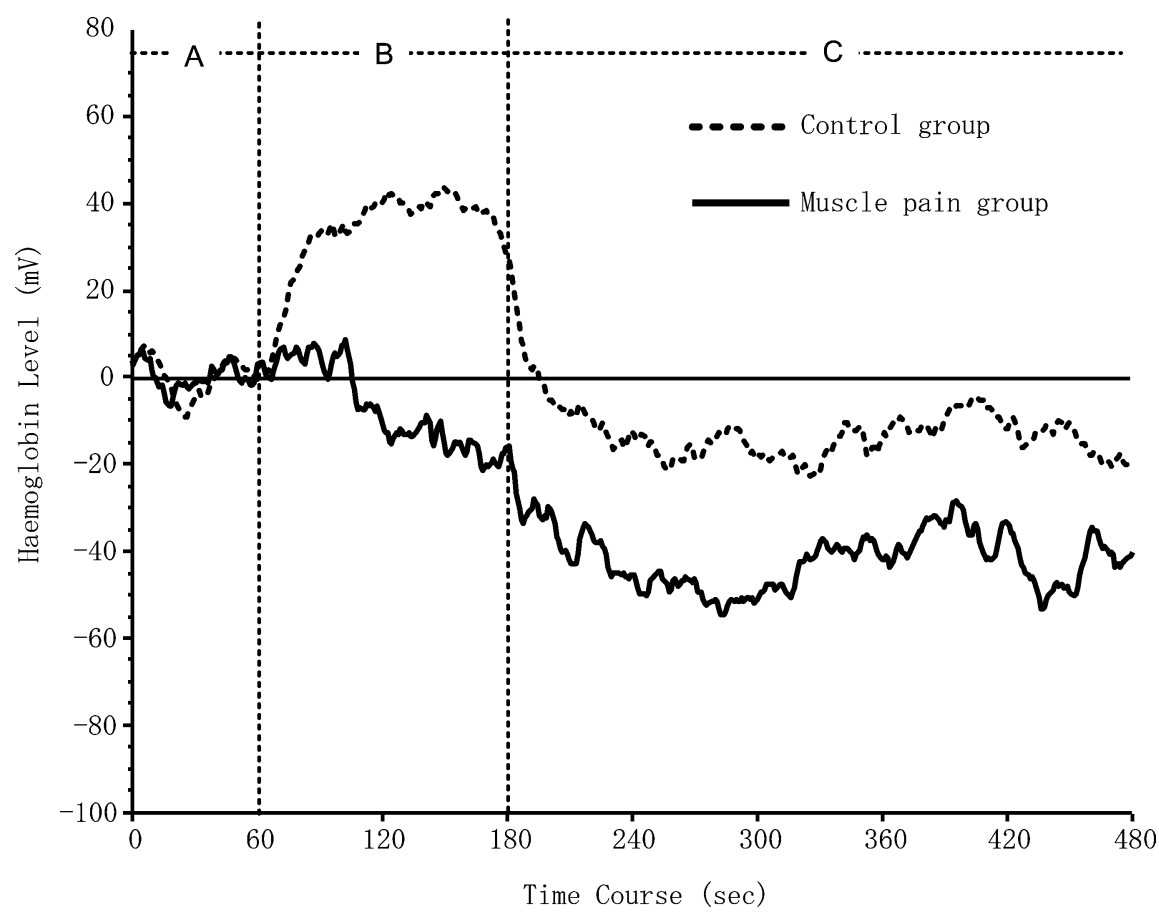

Fig. 5. Haemodynamic responses in chronically painful trapezius muscle to CPS in human subjects. This study evaluated the haemodynamic responses in trapezius muscles to CPS in subjects with localized trapezius myalgia and asymptomatic subjects. Nine male subjects with chronic localized trapezius muscle pain (mean age: 23.2 years) and nine matched controls (mean age: 24.6 years), who had no medical history of migraine, hypertension or sustained pain in the trapezius region participated. Haemoglobin $(\mathrm{Hb})$ levels were continuously recorded 1 minute before, 2 minutes during, and 5 minutes after CPS using NIR spectroscopy. Results showed that the muscle pain subjects exhibited a significantly lower mean $\mathrm{Hb}$ level than non-muscle pain subjects during CPS ( $p=0.0367)$. A: Baseline (1 min), B: during CPS ( $2 \mathrm{~min})$, C: recovery phase (5 min). According to [24].

\section{Acknowledgement}

These researches were done by Grant-in-aids from the Ministry of Education, Sciences, Sports, Cultures in Japan (\#08457528, \#12671883,\#12557170, \#13877327).

\section{References}

[1] A. Wänman, The relationship between muscle tenderness and craniomandibular disorders: a study of 35-year-olds from the general population, J. Orofac. Pain 9 (1995), 235-243.

[2] J.C. Türp, J.C. Kowalski, N. O'Leary and C.S. Stohler, Pain maps from facial pain patients indicate a broad pain geography, J. Dental Res. 77 (1998), 1465-1472.

[3] K. Maekawa, G.T. Clark and T. Kuboki, Intramuscular hypoperfusions, adrenergic receptors, and chronic muscle pain, J. Pain 3 (2002), 251-260.

[4] B. Chance, Rapid and sensitive spectrophotometry: III. A double beam apparatus, Rev. Sci. Instrum. 22 (1951), $634-638$.

[5] B. Chance, M.T. Dait, C. Zhang, T. Hamaoka and F. Hagerman, Recovery from exercise-induced desaturation in elite competitive rowers, Am. J. Physiol. 262 (1992), C766-C775.

[6] D.S. Smith, W. Levy, M. Maris and B. Chance, Reperfusion hyperoxia in brain after circulatory arrest in humans, Anesthesiology 73 (1990), 12-19.

[7] Y.J. Kim, T. Kuboki, Y. Tsukiyama, K. Koyano and G.T. Clark, Haemodynamic changes in human masseter and temporalis muscle induced by different levels of isometric contractions, Arch. Oral Biol. 44 (1999), 641-650. 
[8] G. Sjøgaard, B. Keins, K. Jorgensen and B. Saltin, Intramuscular pressure, EMG and blood flow during low level prolonged static contraction in man, Acta Physiol. Scand. 128 (1986), 475-484.

[9] K. Maekawa, T. Kuboki, G.T. Clark, M. Shinoda and A. Yamashita, Cold pressor stimulus temperature and resting masseter muscle haemodynamics in normal human subjects, Arch. Oral Biol. 43 (1998), 849-859.

[10] E.E. Blaak, M.A. van Dark, G.J. Kemerink, M.T. Pakbiers, G.A. Heidendal and W.H. Saris, Total forearm blood flow as an indicator of skeletal muscle blood flow: Effect of subcutaneous adipose tissue blood flow, Clin. Sci. (Lond.) 87 (1994), $559-566$.

[11] M.C. Koss, Characterization of adrenoceptor subtypes in cat cutaneous vasculature, J. Pharmacol. Exp. Ther. 254 (1990), 221-227.

[12] L.E. Lindblad, L. Ekenvall, B.M. Etzell and S. Bevegård, Adrenoceptors in Raynaud's disease, J. Cardiovasc. Pharmacol. 14 (1989), 881-885.

[13] L.K. Fotedar, J.A. Slopis, P.A. Narayama, M.J. Fenstermacher, J. Pivarnik and I.J. Butler, Proton magnetic resonance of exercise-induced water changes in gastrocnemis muscle, J. Applied Physiol. 5 (1990), 1695-1701.

[14] J.A. Detre, J.S. Leigh, D.S. Williams and A.P. Koretsky, Perfusion imaging, Magn. Reson. Med. 23 (1992), 37-45.

[15] K.K. Kwong, J.W. Belliveau, D.A. Chesler, I.E. Goldberg, R.M. Weisskoff, B.P. Poncelet et al., Dynamic magnetic resonance imaging of human brain activity during primary sensory stimulation, Proc. Natl. Acad. Sci. USA 89 (1992), 56755679.

[16] J.L. Fleckenstein, R.C. Canby, R.W. Parkey and R.M. Peshock, Acute effects of exercise on MR imaging of skeletal muscle in normal volunteers, AJR Am. J. Roentgenol. 151 (1988), 231-237.

[17] F.G. Shellock, T. Fukunaga, J.H. Mink and V.R. Edgerton, Acute effects of exercise on MR imaging of skeletal muscle: Concentric vs eccentric actions, AJR Am. J. Roentgenol. 156 (1991), 765-768.

[18] J.A. Jeneson, J.S. Taylor, D.B. Vigneron, T.S. Willard, L. Carvajal, S.J. Nelson et al., ${ }^{1} \mathrm{H}$ MR imaging of anatomical compartments within the finger flexor muscles of the human forearm, Magn. Reson. Med. 15 (1990), 491-496.

[19] E.R. Weidman, H.C. Charles, R. Negro-Vilar, M.J. Sullivan and J.R. MacFall, Muscle activity localization with ${ }^{31} \mathrm{P}$ spectroscopy and calculated $\mathrm{T}_{2}$-weighted ${ }^{1} \mathrm{H}$ images, Invest. Radiol. 26 (1991), 309-316.

[20] B.T. Archer, J.L. Fleckenstein, L.A. Bertocci, R.G. Haller, B. Barker, R.W. Parkey et al., Effect of perfusion on exercised muscle; MR imaging evaluation, J. Magn. Reson. Imaging 2 (1992), 407-413.

[21] Y. Gan, T. Sasai, H. Nishiyama, X. Ma, Z. Zhang and H. Fuchihata, Magnetic resonance imaging of human mandibular elevator muscles after repetitive maximal clenching exercise, Arch. Oral Biol. 45 (2000), 247-251.

[22] T. Kuboki, K. Suzuki, K. Maekawa, M. Inoue-Minakuchi, C.O. Acero Jr, Y. Yanagi, T. Wakasa, K. Kishi, H. Yatani and G.T. Clark, Correlation of the near-infrared spectroscopy signals with signal intensity in $\mathrm{T}_{2}$-weighted magnetic resonance imaging of the human masseter muscle, Arch. Oral Biol. 46 (2001), 721-727.

[23] M. Inoue-Minakuchi, T. Kuboki, K. Maekawa, Y. Yanagi, E. Inoue, T. Wakasa, K. Kishi, H. Yatani and G.T. Clark, Signal intensity changes in T2-weighted MR image of the human trapezius muscle upon cold pressor stimulation, Dentomaxillofac. Radiol. 31 (2002), 350-354.

[24] C.O. Acero Jr, T. Kuboki, K. Maekawa, A. Yamashita and G.T. Clark, Haemodynamic responses in chronically painful, human trapezius muscle to cold pressor stimulation, Arch. Oral Biol. 44 (1999), 805-812. 


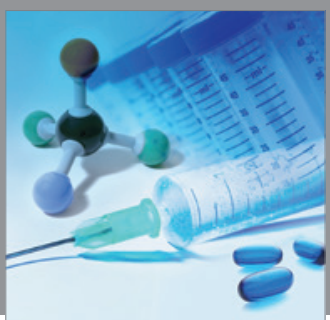

International Journal of

Medicinal Chemistry

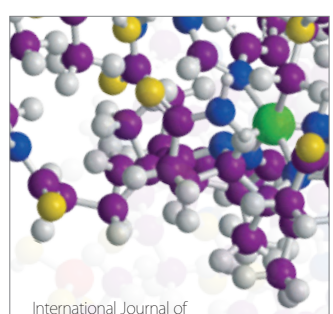

Carbohydrate Chemistry

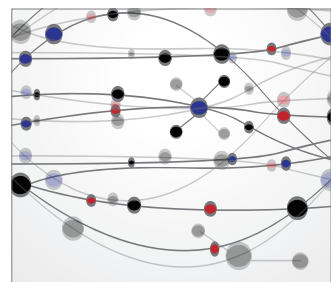

The Scientific World Journal
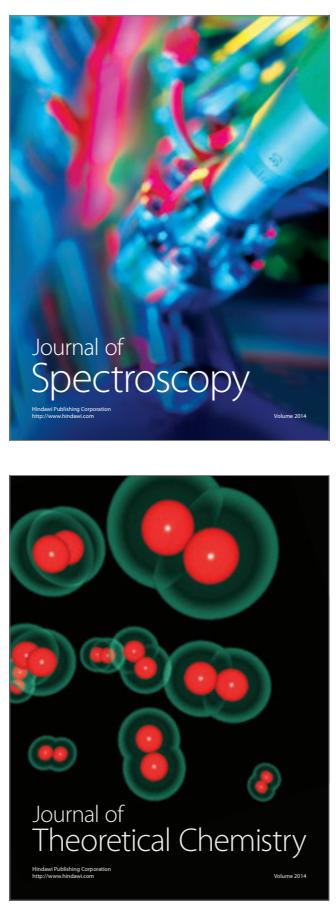
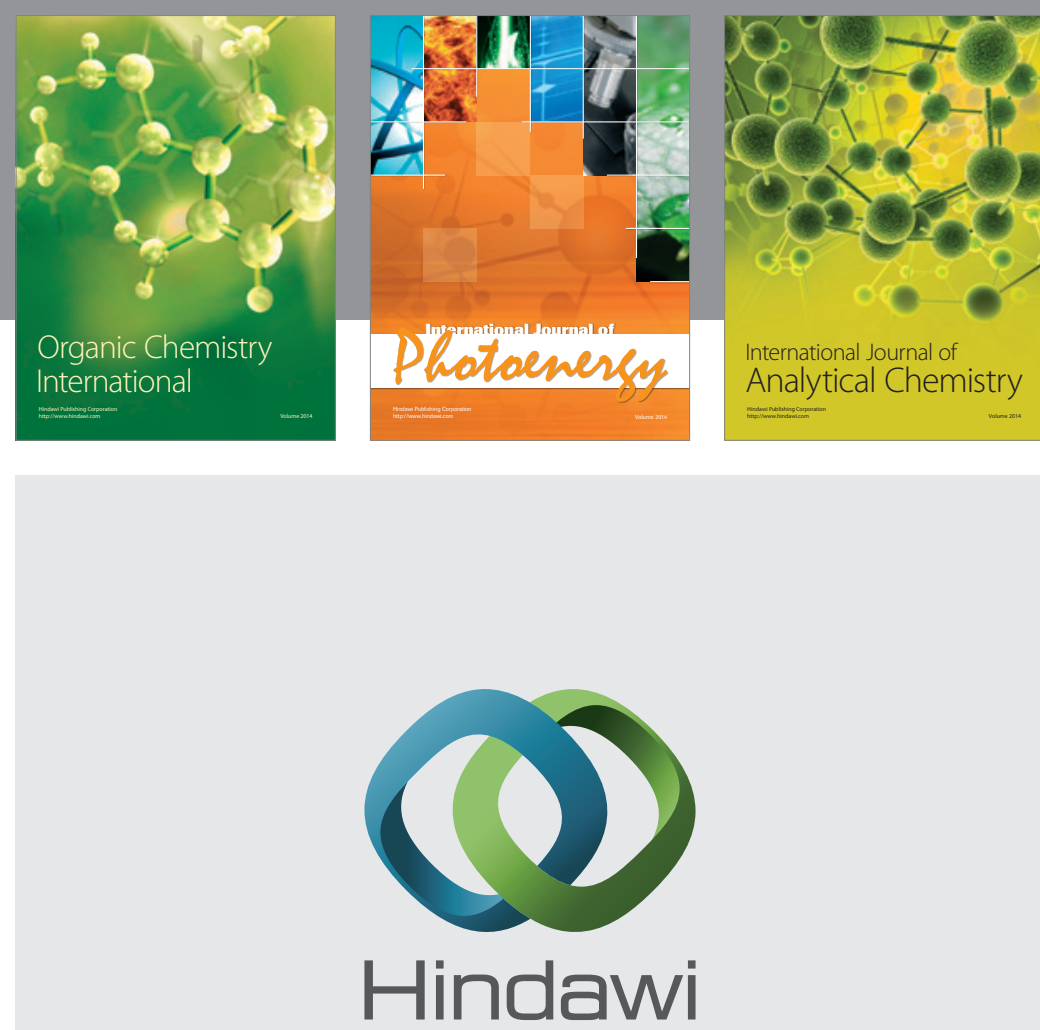

Submit your manuscripts at

http://www.hindawi.com
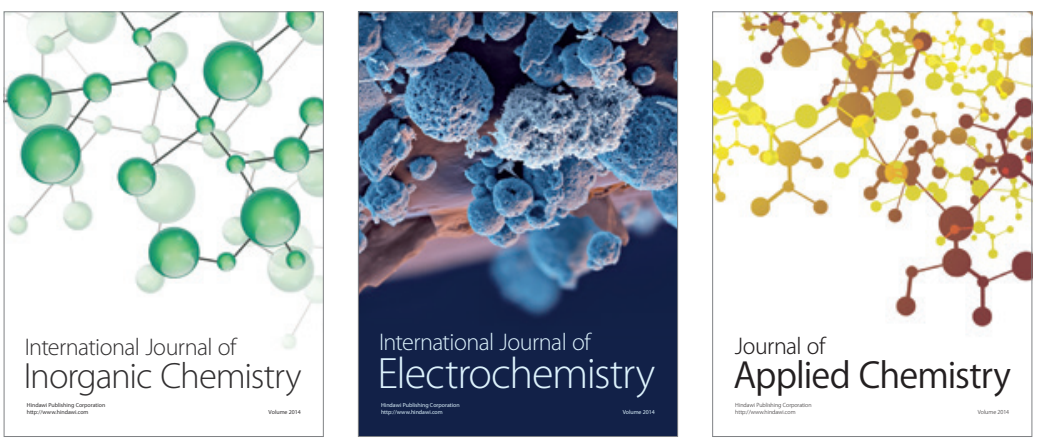

Journal of

Applied Chemistry
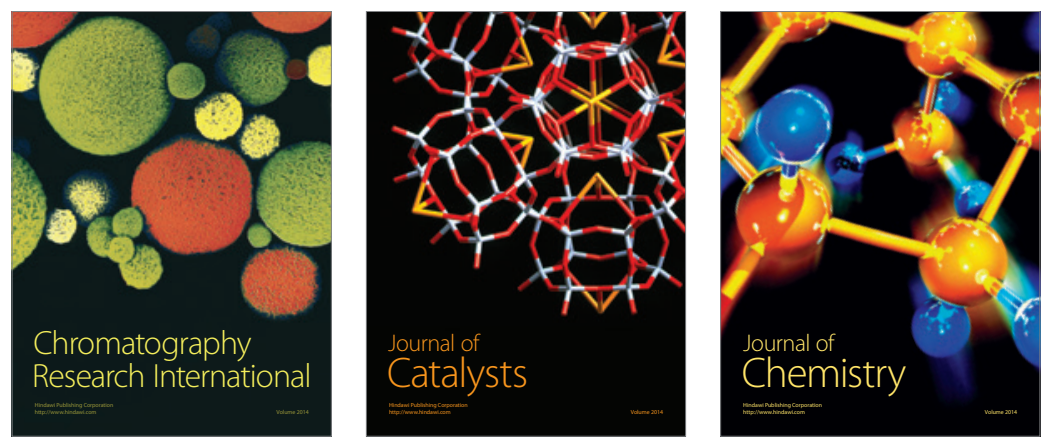
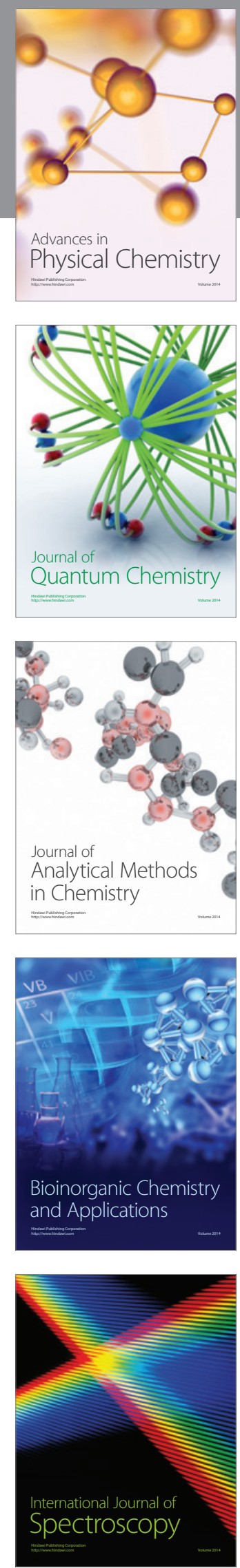